\title{
Acesso às consultas médicas nos serviços públicos de saúde
}

\author{
Access to medical appointments in public health services \\ El acceso a las consultas médicas en los servicios de salud pública
}

\author{
Orlene Veloso Dias, Flávia Ferreira Araújo, Rayana Meireles de Oliveira, Rosângela Barbosa Chagas, Simone de Melo Costa
}

\section{Resumo}

Objetivo: Avaliar o acesso às consultas médicas em serviços públicos de saúde. Métodos: Pesquisa transversal, analítica de base populacional, em município de porte médio, no estado de Minas Gerais, Brasil. Os dados foram coletados a partir de questionário semiestruturado a uma amostra representativa do município, de 2150 indivíduos. $O$ tratamento estatístico considerou o nível de significância $p<0,05$. Resultados: Dos entrevistados, $19,4 \%$ afirmaram ter procurado um profissional/serviço público de saúde nos 15 dias anteriores à entrevista. Entre esses, 75,6\% foram na Atenção Primária à Saúde (APS). A maior procura foi por parte do sexo feminino $(p<0,001)$ e os sem plano de saúde $(p<0,001)$. A maioria $(77,2 \%)$ foi atendida na primeira vez que procurou o serviço de saúde pública. Pode-se destacar uma maior prevalência ao não acesso quando a procura foi na APS e para tratamento/reabilitação $(p<0,05)$. A utilização de consultas médicas no ano foi observada para $54,4 \%$, com maiores médias para mulheres, adultos e sem plano $(p<0,05)$. Conclusão: Necessita-se repensar a gestão de saúde pública e as práticas dos profissionais para efetivar o acesso às consultas médicas. Assim, sugere-se adotar medidas de acolhimento e educação permanente junto aos profissionais da saúde da família.

\section{Abstract}

Objective: To assess the accessibility to medical appointments in public health services. Methods: Transversal, analytic, population-based research in a medium-sized city in the state of Minas Gerais, Brazil. The data were collected from a semi structured questionnaire presented to a representative sample of the city, of 2150 people. The statistic treatment considered $\mathrm{p}<0.05$ as the level of significance. Results: $19.4 \%$ of the interviewees reported having sought a public health professional/service up to 15 days prior to the interview. Among these cases, $75.6 \%$ occurred in the Primary Health Care $(\mathrm{PHC})$. The majority of these seekers were females $(\mathrm{p}<0.001)$ and people with no private health insurance $(p<0.001)$. The majority $(77.2 \%)$ was seen in the first time they sought the public health service. A bigger prevalence of no access could be highlighted when the patient sought the PHC and for treatment/rehabilitation $(p<0.05)$. The use of medical service in that year was reported to be $54.4 \%$, with a higher average for women, adults and people with no private health insurance $(\mathrm{p}<0.05)$. Conclusion: It is necessary to think over the public health management and professional conduct to make medical appointments effective. Therefore, adopting welcoming and permanent teaching measures among family health professionals is advised.

\section{Palavras-chave:}

Acesso aos Serviços de Saúde Visitas a Consultório Médico Comportamento do Consumidor Sistema Único de Saúde

Keywords:

Access to Health Services Visits to Medical Offices Consumer Behavior Public Health System
Como citar: Dias OV, Araújo FF, Oliveira RM, Chagas RB, Costa SM. Acesso às consultas médicas nos serviços públicos de saúde. Rev Bras Med Fam Comunidade. 2016;11(38):1-13. http://dx.doi.org/10.5712/rbmfc11(38)1185
Fonte de financiamento: Ao Ministério da Saúde e Fundação de Amparo à Pesquisa do Estado de Minas Gerais - Fapemig, processo №: CDS-APQ 03616-12, pelo apoio financeiro Parecer CEP: 153.234 (Unimontes), aprovado em 25/11/2012.

Conflito de interesses: declaram não haver.

Procedência e revisão por pares: revisado por pares.

Recebido em: 28/07/2015.

Aprovado em: 03/11/2016. 


\section{Resumen}

Objetivo: Evaluar el acceso a las consultas médicas en los servicios públicos de la salud. Métodos: Investigación transversal, analítica de base poblacional en un municipio de porte medio, en el estado de Minas Gerais, Brasil. Los datos fueron recolectados a partir de cuestionario semiestructurado a una muestra representativa del municipio, de 2150 individuos. El tratamiento estadístico considero el nivel de significación $p<0,05$. Resultados: De los entrevistados, 19,4\% afirmaron haber buscado un profesional/servicio público de salud, en los 15 días anteriores a la entrevista. Entre estos, $75,6 \%$ fueron en la Atención Primaria a la Salud (APS). La mayor búsqueda fue del sexo femenino $(p<0,001)$ y los que no tienen seguro de salud $(p<0,001)$. La mayoría $(77,2 \%)$ fue atendida la primera vez que buscó el servicio de salud pública. Se puede destacar una mayor prevalencia al no acceso cuando la búsqueda fue en el APS y para tratamiento/rehabilitación $(p<0,05)$. La utilización de consultas médicas en el año fue observada para $54,4 \%$, con mayores promedios para mujeres, adultos y los que no tienen seguro $(p<0,05)$. Conclusión: Se necesita volver a pensar la gestión de la salud pública y las prácticas de los profesionales para efectuar el acceso a las consultas médicas. Así, se sugiere adoptar medidas de recepción y educación permanente junto a los profesionales de la salud de la familia.
Palabras clave:

Acceso a los Servicios

de Salud

Visitas a las consultas médicas

Comportamiento del

Consumidor

Sistema Único de Salud

\section{Introdução}

A implantação do Sistema Único de Saúde (SUS) representou um avanço na saúde pública brasileira, embora o país ainda vivencie dificuldades para garantir o amplo acesso da população aos serviços de saúde..$^{1,2}$ Assim, os dados divulgados a partir da Pesquisa Nacional por Amostra de Domicílios (PNAD) revelaram que o acesso aos serviços de saúde aumentou, entretanto, ainda existem desigualdades geográficas e sociais. ${ }^{3}$

O termo acesso, relacionado aos serviços de saúde, pode ser entendido como a "porta de entrada", ou seja, o espaço para acolher o usuário no momento de sua necessidade. ${ }^{4} \mathrm{O}$ acesso relaciona-se ao grau de facilidade ou dificuldade com que os indivíduos obtêm os serviços. Sendo assim, o acesso reflete as características do sistema de saúde, que podem aumentar ou diminuir obstáculos à obtenção dos serviços pela população. ${ }^{5}$

Com relação ao conceito utilização dos serviços de saúde, compreende-se como todo contato direto em consultas médicas e hospitalizações ou mesmo indireto, nos exames preventivos e diagnósticos. $O$ processo de utilização dos serviços é determinado pelo indivíduo que procura o sistema de saúde, pelo profissional que o conduz dentro do sistema e pelas características organizacionais do serviço. ${ }^{6}$

Nesse sentido, o objetivo deste estudo foi avaliar o acesso às consultas médicas em serviços públicos de saúde.

\section{Material e Métodos}

Trata-se de estudo transversal, analítico e de base populacional. Os resultados deste estudo se referem a um recorte de um estudo maior sobre vigilância das doenças crônicas não transmissíveis, realizado em Montes Claros, MG, Brasil, entre agosto de 2010 e julho de 2011, em parceria da Secretaria Municipal de Saúde e Universidade Estadual de Montes Claros (Unimontes). O município é de porte médio, com população estimada, em 2010, de 363.915 habitantes, considerado o polo da macrorregião do estado de MG.

Todos os princípios éticos foram considerados no desenvolvimento da pesquisa e os participantes assinaram o Termo de Consentimento Livre e Esclarecido. Para este recorte da pesquisa, utilizou-se parte 
do banco de dados do estudo maior, após aprovação pelo Comitê de Ética em Pesquisa da Universidade Estadual de Montes Claros (Unimontes), parecer № 153.234.

Para definir o tamanho da amostra, considerou-se a população com idade igual ou superior a 18 anos e uma prevalência estimada de 50\%, nível de confiança 95\% e margem de erro amostral 3\%. Como a amostragem foi complexa, por conglomerados, setores censitários e domicílios, multiplicou-se o tamanho da amostra pelo fator de correção (deff) de 2,0, acrescido de $10 \%$ para compensar perdas. O tamanho da amostra estimada foi de 2150 indivíduos, entre adultos e idosos.

$\mathrm{Na}$ alocação da amostra, sortearam-se 43 setores censitários, sendo 40 urbanos e 3 rurais. Em cada setor sortearam-se 50 domicílios, e nesses entrevistou-se apenas um dos moradores. No caso de ausência dos moradores, no momento da pesquisa, três novas visitas foram efetuadas, antes de substituir pelo próximo domicilio à direita ou subsequentes. Realizou-se 1 piloto, com 20 indivíduos, para adequações metodológicas, buscando antecipar situações possíveis no processo da coleta de dados. Dados do piloto não compuseram o estudo final.

O instrumento de coleta de dados embasou-se no questionário de monitoramento de fatores de risco para doenças crônicas, Vigilância de Fatores de Risco e Proteção para Doenças Crônicas por Inquérito Telefônico (Vigitel), ${ }^{7}$ entretanto, no atual estudo o instrumento foi aplicado pelo contato pessoal direto, ou seja, presencial.

As variáveis dependentes foram: atendimento na primeira vez em que procurou por um serviço de saúde pública nos 15 dias anteriores à entrevista e média de consultas médicas efetuadas no setor público nos últimos 12 meses. E as variáveis independentes foram: sexo, faixa etária, estado civil, cor da pele autodeclarada, escolaridade, ter plano de saúde, pontos de atenção no SUS, motivo da procura pelos serviços públicos de saúde, ter algo que desagrada no serviço público, recomendar o profissional público e autopercepção de saúde.

O tratamento estatístico envolveu a análise descritiva de valores percentuais, médias e desvio padrão. As médias de consultas médicas nos últimos 12 meses foram comparadas conforme os grupos formados pelas variáveis independentes, pelos testes $t$ student e Anova, com adoção do teste de Bonferroni para distinguir as diferenças entre as múltiplas comparações. Foi efetuada a análise bivariada, pelo teste qui quadrado de Pearson, para associar a procura pelos serviços de saúde pública, nos 15 dias anteriores à pesquisa, conforme sexo e condição de ter plano de saúde.

Em seguida, realizou-se a análise bivariada, regressão de Poisson com variância robusta, entre atendimento na primeira vez que procurou o serviço público de saúde e variáveis independentes. Após essa análise, todas as variáveis independentes com associação até o nível de $20 \%$ foram para análise múltipla, com exclusão progressiva das variáveis com maior valor de $p$ até a permanência daquelas com $p<0,05$.

A significância estatística foi determinada pelo teste de Wald, estimando-se as razões de prevalências ajustadas e respectivos intervalos de $95 \%$ de confiança, considerando os ajustes pelo efeito do desenho (peso de ponderação). Assim, todo tratamento estatístico envolveu o uso de peso de amostragem, a partir das unidades amostrais primárias (setores censitários) e secundárias (domicílios), para compensar as probabilidades desiguais de seleção entre os elementos da amostra. ${ }^{8,9}$ Os dados foram processados no software IBM SPSS ${ }^{\circledR}$ versão 22.0 . 


\section{Resultados}

Participaram 2150 pessoas, sendo 79,5\% adultos (18 a 59 anos) e 20,5\% idosos (60 anos ou mais), $63,3 \%$ do sexo feminino. A cor de pele autodeclarada foi branca para $26,1 \%$, preta para $12,9 \%$, amarela $2,5 \%$, parda $58,2 \%$ e indígena para $0,3 \%$. A condição de ter plano de saúde foi relatada por $36,3 \%$. Quanto ao atendimento ofertado nas Unidades do serviço de saúde pública, 64,2\% informaram que há algo que os desagrada. Entre os desagrados destacam-se: dificuldade de marcar consultas no geral $(18,2 \%)$, de marcar exames laboratoriais e outros exames (14,5\%), de marcar consulta com especialistas (17,0\%), de acesso geográfico ao local de atendimento $(0,9 \%)$, demora no atendimento $(32,9 \%)$ e outros desagrados (16,5\%). A autopercepção de saúde geral foi constatada para $29,7 \%$ dos participantes como regular/ruim.

Dos entrevistados, 19,4\% (416) afirmaram ter procurado um profissional/serviço público de saúde, nos 15 dias que antecederam à realização da entrevista. Entre esses indivíduos, o tratamento de doença foi o motivo mais destacado $(41,7 \%)$. Em relação aos diferentes níveis de atenção da saúde, no âmbito público, a procura por serviços foi, majoritariamente, na Atenção Primária à Saúde $(75,6 \%)$, seguida de hospital $(15,6 \%)$ e clínicas especializadas (8,9\%). Na Tabela 1 observa-se que a procura foi em maior percentual no sexo feminino $(p<0,001)$ e entre as pessoas sem plano de saúde $(p<0,001)$.

Tabela 1. Distribuição dos pesquisados quanto à procura de serviço público de saúde nas duas semanas anteriores à entrevista segundo sexo e plano de saúde.

\begin{tabular}{lccc}
\hline \multirow{2}{*}{ Variáveis } & \multicolumn{2}{c}{ Procurou por um serviço público de saúde nas duas últimas semanas? } \\
\cline { 2 - 4 } & Sim & Não & p \\
\hline Sexo & & & 0,001 \\
\hline Feminino & $21,9 \%$ & $78,1 \%$ & $84,9 \%$ \\
Masculino & $15,1 \%$ & & $p<0,001$ \\
\hline Plano de Saúde & & $87,5 \%$ & \\
\hline Sim & $12,5 \%$ & $76,3 \%$ & \\
Não & $23,7 \%$ & & \\
\hline
\end{tabular}

* valor percentual ajustado pelo efeito do desenho.

A maioria das pessoas foi atendida na primeira tentativa por procura de serviço de saúde pública, nos últimos 15 dias $(77,2 \%)$. A análise bivariada demonstra maior prevalência de não acesso pelo sexo feminino $(R P=1,03)$; entre aqueles sem escolaridade $(R P=1,12)$; os que não possuem união estável/casados $(R P=1,04)$, entre os idosos ( $R P=1,05)$; os que se autodeclararam negros $(R P=1,04)$; entre os que procuraram os serviços da Atenção Primária à Saúde (APS), no SUS (RP=1,15); os que manifestaram algum desagrado com os profissionais/serviços públicos de saúde procurados ( $R P=1,23)$; os que não recomendariam o profissional/serviço de saúde pública $(R P=1,43)$ e entre aqueles que autoavaliaram a sua saúde geral como regular/ruim $(\mathrm{RP}=1,03)$ (Tabela 2$)$.

Após o ajuste entre as variáveis, pelo modelo múltiplo, verificou-se que o não atendimento pela primeira vez que procurou o serviço público ficou associado com as pessoas sem união estável/casado $(R P=1,11)$, procura na Atenção Primária à Saúde $(R P=1,22)$, para tratamento ou reabilitação $(R P=1,21)$, entre os que afirmaram ter algo que os desagradam no profissional/serviço público $(R P=1,19)$, os que não recomendariam o profissional/serviço público de saúde $(R P=1,27)$ e entre os que autoavaliaram a sua saúde geral como regular/ruim $(\mathrm{RP}=1,12)$ (Tabela 2$)$. 
Tabela 2. Análise bivariada e múltipla do acesso à assistência em saúde na primeira vez que procurou o serviço público, nos 15 dias anteriores, conforme perfil sociodemográfico, serviços de saúde e autoavaliação da saúde.

\begin{tabular}{|c|c|c|c|c|}
\hline \multirow{2}{*}{$\begin{array}{l}\text { Variáveis } \\
\text { Perfil }\end{array}$} & \multicolumn{2}{|c|}{$\begin{array}{l}\text { Acesso à assistência em saúde entre os que } \\
\text { procuraram o serviço público, nos últimos } 15 \\
\text { dias }(n=416)\end{array}$} & \multicolumn{2}{|c|}{$\begin{array}{l}\text { Razão de Prevalência - RP (IC95\%)* } \\
\text { Valor de p }\end{array}$} \\
\hline & $\begin{array}{c}n=321 \\
\operatorname{Sim}(\%)^{*}\end{array}$ & $\begin{array}{c}n=95 \\
\text { Não }(\%)^{*}\end{array}$ & $\begin{array}{c}\text { Bivariada } \\
\mathbf{p}\end{array}$ & $\begin{array}{c}\text { Múltipla } \\
\text { p }\end{array}$ \\
\hline \multicolumn{5}{|l|}{ Sexo } \\
\hline Masculino & 80,4 & 19,6 & 1 & - \\
\hline Feminino & 76,0 & 24,0 & $1,03(1,01-1,06) p=0,006$ & \\
\hline \multicolumn{5}{|l|}{ Escolaridade } \\
\hline Superior & 81,9 & 18,1 & 1 & - \\
\hline Não superior & 78,9 & 21,1 & $1,03(0,99-1,06) p=0,127$ & \\
\hline Analfabeto & 67,8 & 32,2 & $1,12(1,06-1,18) p<0,001$ & \\
\hline \multicolumn{5}{|l|}{ Estado Civil } \\
\hline União estável/casado & 79,6 & 20,4 & 1 & 1 \\
\hline Outros & 75,1 & 24,9 & $1,04(1,01-1,08) p=0,033$ & $1,11(1,02-1,21) p=0,016$ \\
\hline \multicolumn{5}{|l|}{ Faixa etária } \\
\hline 60 anos e mais & 72,5 & 27,5 & $1,05(1,02-1,08) p<0,001$ & - \\
\hline 18 a 59 anos & 78,7 & 21,3 & 1 & \\
\hline \multicolumn{5}{|l|}{ Cor de pele } \\
\hline Não preta & 78,0 & 22,0 & 1 & - \\
\hline Preta & 72,4 & 27,6 & $1,04(1,01-1,09) p=0,017$ & \\
\hline Serviços de saúde & $\begin{array}{c}n=321 \\
\operatorname{Sim}(\%)^{*}\end{array}$ & $\begin{array}{c}\mathrm{n}=95 \\
\text { Não }(\%)^{*}\end{array}$ & $\begin{array}{c}\text { Bivariada } \\
\mathbf{p}\end{array}$ & $\begin{array}{c}\text { Múltipla } \\
\text { p }\end{array}$ \\
\hline \multicolumn{5}{|l|}{ Plano de saúde } \\
\hline Sim & 75,8 & 24,2 & $1,02(0,99-1,05) p=0,099$ & - \\
\hline Não & 78,3 & 21,7 & 1 & \\
\hline \multicolumn{5}{|l|}{ Pontos de Atenção do SUS } \\
\hline Atenção Primária & 73,8 & 26,2 & $1,15(1,11-1,18) p<0,001$ & $1,22(1,10-1,35) p<0,001$ \\
\hline Outro & 90,0 & 10,0 & 1 & 1 \\
\hline \multicolumn{5}{|l|}{ Motivo procura } \\
\hline Exames/consulta & 81,3 & 18,7 & 1 & 1 \\
\hline Tratamento/reabilitação & 75,7 & 24,3 & $1,04(0,99-1,10) p=0,075$ & $1,21(1,14-1,30) p<0,001$ \\
\hline \multicolumn{5}{|c|}{$\begin{array}{l}\text { Desagrado com profissional/serviço } \\
\text { público de saúde }\end{array}$} \\
\hline Sim & 69,3 & 30,7 & $1,23(1,20-1,27) p<0,001$ & $1,19(1,10-1,28) p<0,001$ \\
\hline Não & 94,1 & 5,9 & 1 & 1 \\
\hline \multicolumn{5}{|c|}{$\begin{array}{l}\text { Recomenda o profissional/serviço } \\
\text { público de saúde }\end{array}$} \\
\hline Sim & 88,7 & 11,3 & 1 & 1 \\
\hline Não & 45,7 & 54,3 & $1,43(1,35-1,43) p<0,001$ & $1,27(1,18-1,35) p<0,001$ \\
\hline Autoavaliação da saúde & $\begin{array}{c}n=321 \\
\operatorname{Sim}(\%)^{*}\end{array}$ & $\begin{array}{c}n=95 \\
\text { Não (\%)* }\end{array}$ & $\begin{array}{c}\text { Bivariada } \\
\mathbf{p}\end{array}$ & $\begin{array}{l}\text { Múltipla } \\
\text { p }\end{array}$ \\
\hline \multicolumn{5}{|l|}{ Percepção da saúde geral } \\
\hline Muito bom/bom & 78,4 & 21,6 & 1 & 1 \\
\hline Regular/ruim & 75,1 & 24,9 & $1,03(1,00-1,05) p=0,050$ & $1,12(1,03-1,22) p=0,007$ \\
\hline
\end{tabular}

* valor percentual e razão de prevalência (Intervalo de Confiança 95\%) ajustados pelo efeito do desenho. 
Ao levar em consideração a utilização do serviço público de saúde, especificamente as consultas médicas, no ano anterior à pesquisa, para toda população pesquisada ( $n=2150$ ) verificou-se que $54,4 \%$ consultaram pelo menos uma vez. A variação de consultas foi de zero a 30 ao ano, sendo a média ( \pm desvio padrão) de 1,56 $( \pm 2,64)$ consultas por indivíduo.

Ao comparar as médias de consultas entre os grupos, constatou-se maior média no sexo feminino, entre os com maior nível de escolaridade, entre os adultos, para os que não possuem plano de saúde, informaram não haver desagrados em relação ao profissional/setor público, não recomendariam o profissional/setor público e, para os que consideraram sua saúde geral como regular/ruim (Tabela 3).

Tabela 3. Média e desvio padrão do número de consultas médicas no setor público conforme perfil sociodemográfico, serviços de saúde e autoavaliação da saúde. Adultos e idosos de Montes Claros, MG, Brasil.

\begin{tabular}{|c|c|c|c|}
\hline \multirow{2}{*}{$\begin{array}{l}\text { Variáveis } \\
\text { Perfil } \\
\end{array}$} & \multicolumn{2}{|c|}{ Consultas médicas nos últimos 12 meses } & \multirow{2}{*}{$\begin{array}{c}\text { Teste } \boldsymbol{t} \text { (student) } \\
\text { Anova (Bonferroni) }^{\star *} \\
p\end{array}$} \\
\hline & Média* & Desvio padrão* & \\
\hline \multicolumn{4}{|l|}{ Sexo } \\
\hline Masculino & 1,30 & 2,474 & \multirow[t]{2}{*}{$p<0,001$} \\
\hline Feminino & 1,71 & 2,723 & \\
\hline \multicolumn{4}{|l|}{ Escolaridade } \\
\hline Superior & 1,72 & 3,547 & \multirow{3}{*}{$\begin{array}{c}\mathrm{p}<0,001 \text { para todas as } \\
\text { comparações }^{\star \star}\end{array}$} \\
\hline Não superior & 1,59 & 2,547 & \\
\hline Analfabeto & 1,36 & 1,480 & \\
\hline \multicolumn{4}{|l|}{ Faixa etária } \\
\hline 60 anos e mais & 1,51 & 2,392 & \multirow[t]{2}{*}{$p=0,001$} \\
\hline 18 a 59 anos & 1,57 & 2,701 & \\
\hline Serviços de saúde & Média* & Desvio padrão* & $\mathbf{p}$ \\
\hline \multicolumn{4}{|l|}{ Plano de saúde } \\
\hline Sim & 1,02 & 2,302 & \multirow[t]{2}{*}{$p<0,001$} \\
\hline Não & 1,89 & 2,779 & \\
\hline \multicolumn{4}{|l|}{ Ponto de Atenção no SUS*** } \\
\hline Atenção Primária & 1,52 & 2,564 & \multirow[t]{2}{*}{$p=0,003$} \\
\hline Outro & 1,65 & 2,944 & \\
\hline \multicolumn{4}{|c|}{ Desagrado com o profissional/serviço público de saúde } \\
\hline Sim & 1,52 & 2,533 & \multirow[t]{2}{*}{$p<0,001$} \\
\hline Não & 1,60 & 2,810 & \\
\hline \multicolumn{4}{|c|}{ Recomenda o profissional/serviço público de saúde ${ }^{\star * *}$} \\
\hline Sim & 1,40 & 2,602 & \multirow[t]{2}{*}{$\mathrm{p}<0,001$} \\
\hline Não & 1,94 & 2,797 & \\
\hline Avaliação de saúde geral & Média* & Desvio padrão* & $\mathbf{p}$ \\
\hline \multicolumn{4}{|l|}{ Autopercepção de saúde } \\
\hline Muito bom & 0,97 & 1,852 & \multirow{4}{*}{$\begin{array}{c}\mathrm{p}<0,001 \text { para todas as } \\
\text { comparações }\end{array}$} \\
\hline Bom & 1,43 & 2,581 & \\
\hline Regular & 2,12 & 3,055 & \\
\hline Ruim & 2,82 & 3,127 & \\
\hline
\end{tabular}




\section{Discussão}

Entre os 2150 indivíduos adultos e idosos, houve predomínio do sexo feminino, pessoas que se autodeclararam cor de pele parda e sem plano de saúde. Quanto à assistência nos serviços de saúde pública, a maioria manifestou algum desagrado com os profissionais/serviços, sendo destacadas as dificuldades para marcar consultas e exames, além de dificuldade de acesso ao local de assistência e demora no atendimento. Esses resultados corroboram a pesquisa conduzida em Campina Grande, PB, Brasil, com usuários do SUS cadastrados na Estratégia de Saúde da Família, que demonstraram insatisfação com o longo tempo de espera, tanto para marcar consultas como para ser atendido nas consultas agendadas. ${ }^{2}$

No atual estudo, uma parcela importante dos entrevistados procurou um serviço de saúde pública, nos 15 dias anteriores à entrevista, sendo a maior procura pelas mulheres e entre os sem plano de saúde. O maior uso dos serviços de saúde por parte das mulheres, no Brasil, é fato bem conhecido. ${ }^{3}$ Para aqueles que não possuem plano de saúde e recursos financeiros para arcar com as despesas dos serviços privados, o setor público torna-se a real possibilidade de assistência à saúde, direito garantido constitucionalmente, no Brasil.

Esse direito deve ser assegurado pelo Estado, de forma universal. E para isso se faz necessário um modelo social ético e equânime, baseado na inclusão social e na solidariedade humana. A concretização do acesso universal aos serviços de saúde requer luta constante para fortalecer a saúde como um bem público, direito individual e coletivo. ${ }^{10}$

Pesquisa conduzida em 6.797 domicílios, com 11.207 indivíduos, no estado de Minas Gerais apontou que a Estratégia de Saúde da Família (ESF) tornou-se uma via que garante a materialização da equidade no SUS a partir de um organizado processo de trabalho da equipe profissional. Nesse sentido, desenvolver políticas públicas para expansão da ESF com qualidade pode contribuir para potencializar a melhora de indicadores de saúde nos grupos populacionais, por favorecer aos mais pobres o acesso aos serviços de saúde, principalmente na APS. A macrorregião Norte de Minas apresentou a maior taxa de visitação domiciliar por algum profissional de saúde, $93,16 \%,{ }^{11}$ sendo essa a região de localização do município em que foi conduzido o presente estudo.

O fato do maior percentual de pessoas que procuraram o serviço público, nas duas semanas anteriores, não recomendar o profissional/serviço público é preocupante, pois sugere fragilidades no serviço e na assistência recebida. Ainda mais, ao considerar que o principal motivo para a procura foi para tratar alguma doença. A não recomendação do serviço/profissional está relacionada à insatisfação do usuário do SUS. Neste estudo, o percentual dos que recomendariam foi maior entre os satisfeitos com o setor de saúde pública quando comparados aos que manifestaram algum tipo de desagrado.

O local de procura/atendimento no SUS foi em maior percentual nas unidades vinculadas à APS. Uma parcela importante $(22,8 \%)$ não recebeu atendimento na primeira vez que procurou o serviço. Dados de inquérito nacional apresentam percentual menor de pessoas não atendidas pela primeira vez que procuraram o serviço de saúde $(3,8 \%)$, sendo o principal motivo a falta de vaga. ${ }^{3}$

A saúde pública tem como um importante propósito combater as desigualdades na saúde, na busca de equidade no acesso aos cuidados. Mesmo em países desenvolvidos da Europa, especificamente na França, ainda existem desigualdades significativas no acesso a serviços especializados e nos preventivos. ${ }^{12}$ 
Pesquisa que comparou dois municípios, um da Colômbia e outro do Brasil, com 2.163 e 2.155 pessoas, respectivamente, revelou obstáculos no acesso à saúde nos dois países. Na Colômbia, as maiores barreiras dizem respeito ao acesso inicial aos cuidados de saúde e na resolução do problema. No Brasil há insuficiente disponibilidade de unidades de saúde, médicos e medicamentos. ${ }^{13}$

Estudo que incluiu a participação de 882 usuários da ESF, em São Luís, MA, Brasil, constatou dificuldades no acesso e no uso de serviços. O não funcionamento a partir de 18 horas e nos finais de semana foram as principais dificuldades apresentadas, sendo importante fortalecer a participação da comunidade na gestão local da ESF para identificar as necessidades dos usuários. ${ }^{14}$

A partir dos dados da Pesquisa Nacional por Amostra de Domicílios de 2008, no Brasil, investigaram-se 152.233 trabalhadores de 18 a 64 anos, formais, informais e desempregados. A desigualdade no acesso aos serviços de saúde foi detectada, sendo que para os trabalhadores informais e desempregados a dificuldade é maior e o uso dos serviços de saúde é menor, sugerindo necessidade de políticas de saúde para esse contingente populacional. ${ }^{15}$

O não atendimento às pessoas que procuraram pelos serviços na APS vai contra os princípios do SUS, que pregam o acolhimento no nível primário como importante estratégia de reorganização do modelo assistencial.

Nesse sentido, o Núcleo Técnico da Política Nacional de Humanização do Ministério da Saúde em estudo sobre acolhimento nas práticas de produção de saúde esclareceu que o acolhimento implica em uma relação de cidadania e humanizada, de escuta qualificada; ${ }^{16}$ que se relaciona com: vínculo entre usuários e serviços no setor saúde; resolubilidade da assistência e com a adequação dos serviços às demandas dos usuários. ${ }^{17}$

Assim, o acolhimento se caracteriza pela recepção e escuta daqueles que procuram o serviço, de forma a reconhecer as necessidades de saúde dos usuários para então buscar formas de satisfazê-las, por meio de encaminhamentos, deslocamentos e trânsitos pela rede assistencial. ${ }^{18}$ Para além dessa definição, o acolhimento foi visto por profissionais da saúde da família como importante ferramenta de gestão e regulação do acesso às unidades básicas de saúde e como instrumento para construção de ações mais efetivas no setor da saúde, em outro estudo. ${ }^{19}$

Estudo que analisou o acesso aos serviços de saúde pública, na atenção primária, em Recife, PE, Brasil, a partir da visão de profissionais e de usuários, apresentou como principais barreiras o subfinanciamento dos serviços, a insuficiente cobertura da ESF e de profissionais nas equipes. Apesar dos benefícios próprios da implantação da ESF, necessita-se de priorizar a gestão, para investir na estrutura e melhorar a organização dos serviços da rede de saúde. ${ }^{20}$

Na análise múltipla verificou-se que sexo, faixa etária e cor de pele, apesar de associados, na análise bivariada, com a variável dependente "atendimento na primeira vez que procurou por um serviço de saúde pública, nos últimos 15 dias", não permaneceram no modelo múltiplo, demonstrando serem variáveis de confusão. Neste sentido, as características do perfil não influenciaram no acesso aos serviços de saúde. Isso é importante, uma vez que o setor público deve fornecer um atendimento igualitário e universal para todos aqueles que precisam dos serviços, sem discriminação de qualquer espécie. Fato legalmente respaldado pela constituição brasileira. 
Em relação às associações observadas no modelo final ajustado, detectou-se maior prevalência de não atendimento para aqueles sem união estável/casado, os que procuraram por tratamento/reabilitação, os que relataram desagrado com o profissional/serviço público, os que não recomendariam o profissional/ serviço público e os que autoavaliaram a sua saúde geral como regular/ruim.

No condizente ao não atendimento aos que precisaram do serviço para tratamento de doença ou reabilitação, deve-se considerar que certas doenças, quando não tratadas no momento adequado, poderão desencadear problemas mais graves e impactar, negativamente, na saúde do indivíduo. Em consonância a esse resultado, o estudo demonstra que o não atendimento foi associado à autoavaliação de saúde geral como regular/ruim. Sugere-se que o não atendimento, na primeira procura ao serviço de saúde, influenciou nas opiniões dos pesquisados quanto à manifestação de desagrado com o profissional/serviço público e à não recomendação dos mesmos.

No atual estudo, um pouco mais da metade dos participantes efetuou pelo menos uma consulta médica ao longo do ano. Valor menor que outros estudos de base populacional em estados da região sul, como em Santa Catarina, com 2022 adultos, com prevalência para consultas médicas ao ano igual a $76 \%{ }^{21}$ e no Rio Grande do Sul, igual a $72 \% .{ }^{22}$ Cabe destacar que esses dois estudos da região Sul pesquisaram somente adultos, e o atual estudo pesquisou além de adultos os idosos, o que era de se esperar maior prevalência de consultas médicas, no geral, já que o processo de envelhecimento, geralmente, vem acompanhado de uma maior necessidade de cuidados à saúde.

Essa afirmação foi constatada em estudo com idosos, que concluiu que eles são grandes usuários dos serviços de saúde, o que requer organização da rede pública frente a uma alta demanda por procedimentos diagnósticos terapêuticos entre esse grupo populacional. ${ }^{23}$ Assim, no presente estudo, a inclusão de idosos na amostra sugere aumento no número médio de consultas médicas, entretanto, ao comparar a média de consultas médicas entre idosos e adultos, aponta-se um menor número para os idosos.

Na discussão da utilização dos serviços de saúde a literatura demonstra que a utilização dos serviços na rede do SUS é na sua maioria pelas pessoas com menor escolaridade. ${ }^{24}$ Estudo populacional com indivíduos entre 20 a 75 anos, cadastrados na ESF, verificou problemas na acessibilidade organizacional, tais como o longo tempo de espera para a consulta médica. Os indivíduos com menor nível socioeconômico utilizaram mais o serviço. ${ }^{25}$ Contrariamente, no atual estudo, as pessoas com mais anos de estudos, nível superior, consultaram um médico quase duas vezes ao ano, diferentemente daqueles sem escolaridade, que consultaram 1,36 vezes ao ano.

A maior utilização dos serviços de saúde por pessoas com maior escolaridade também foi observada em estudo internacional, que descreveu a relação entre as desigualdades educacionais e a utilização dos serviços de saúde, por pessoas com idade igual ou maior que 50 anos, residentes em 12 países europeus. Nesse, confirmou-se a presença de desigualdade educacional substancial no uso dos serviços; aqueles com maior nível de escolaridade apresentam maiores recursos, seja cognitivo, comunicativo e/ou relacionais, que contribuem para autonomia das escolhas e decisões em saúde. Entretanto, a organização institucional pode modificar a relação de desigualdade devido à menor escolaridade. ${ }^{26}$

Ainda com relação à média de consultas médicas na saúde pública, observou-se que aqueles sem plano de saúde consultaram quase duas vezes ao ano, assim como aqueles que não recomendariam o 
profissional/serviço público. Este achado sugere que as pessoas sem plano ficam limitadas às possibilidades de alternativas de assistência à saúde, e por isto utilizam bastante o serviço público. Contrariamente, aqueles que consultam mais não recomendam o profissional/serviço, o que reforça a hipótese de utilização do serviço público por falta de acesso aos serviços privados ou suplementares. Contudo, aqueles que não relataram desagrado com o profissional/serviço público apresentaram maior média de consultas médicas, sugerindo que o maior acesso aos serviços de saúde relaciona-se com a maior satisfação quanto aos serviços obtidos.

A menor média de consultas médicas no setor público, ao ano, foi para aqueles que avaliaram a sua saúde como muito boa e, a maior média para aqueles que avaliaram como ruim, com quase uma consulta a cada quatro meses. Em outro estudo de base populacional, em município de médio porte do sul do Brasil, também se encontrou maior prevalência de consultas ao ano por aqueles que avaliaram negativamente a sua saúde. ${ }^{20}$

Dessa forma, possuir uma patologia que necessita de acompanhamento profissional pode justificar a maior média de consultas por indivíduos que autoavaliaram a saúde como regular/ruim. Nessa perspectiva, outro estudo ${ }^{27}$ apontou ser a carga de doenças um importante modulador nas diferenças das prevalências de consultas médicas.

Em se tratando de acesso aos serviços no âmbito da ESF, em pesquisa conduzida no mesmo município do atual estudo, constatou-se, entre 241 agentes comunitários de saúde (ACS), que 99,2\% identificam a situação de saúde das famílias e comunicam as informações para os profissionais da equipe de saúde da família. Os ACS orientam as famílias sobre uso dos serviços $(99,6 \%)$ e fazem o encaminhamento e agendamento de consultas e também de exames $(78,8 \%) .^{28}$

Em adição, no município foi constatado que os profissionais da Medicina realizam em média 3,63 consultas domiciliares por semana, prioritariamente para os acamados. ${ }^{29}$ Esses resultados podem explicar, em parte, o maior número de consultas pelos indivíduos que avaliaram negativamente a sua saúde, pois é possível que a ação do ACS como identificador das famílias de risco e articulador entre as demandas da comunidade e a prestação de serviços dos profissionais da ESF tenha contribuído para esse fato.

Cabe destacar a limitação inerente aos estudos transversais, sendo as medições feitas ao mesmo momento, espaço e tempo, não sendo possível distinguir causa-efeito do problema. Outro aspecto referese ao levantamento de dados por meio de entrevista, com possibilidade de viés de informação, controlado pela adoção de um menor tempo, 15 dias, para a questão que se referiu ao acesso na primeira vez que procurou o serviço público.

Considera-se este estudo importante, realizado com dados primários e amostra representativa da população de cidade de porte médio, com contribuições para saúde pública e coletiva e, também para a prática dos profissionais de saúde, ao discutir acesso e utilização dos serviços, no âmbito da saúde pública.

\section{Conclusões}

Nem todos que procuraram por profissionais/serviços de saúde públicos obtiveram acesso imediato, sugerindo que o acesso à saúde, igualitário e universal, ainda é uma meta a ser alcançada. A dificuldade do acesso aos serviços de saúde foi relacionada à procura na atenção primária, para tratamento ou 
reabilitação e entre os que autoavaliaram a sua saúde como regular/ruim. Então, a APS, apesar de ser considerada a porta de entrada na rede SUS, ainda não consegue acolher todos os usuários que procuram por assistência, para resolução do problema ou para referenciá-los a outros níveis de atenção.

O não acesso imediato aos serviços parece influenciar, negativamente, as opiniões quanto à avaliação do profissional/serviço público e na possibilidade de recomendá-los a outras pessoas. Contudo, entre os que não recomendam a saúde pública observou-se maior utilização das consultas médicas/ano, o que sugere falta de autonomia dos usuários do SUS para escolha dos serviços de saúde a utilizar. Assim, os resultados sugerem necessidade de repensar a gestão de saúde pública e as práticas dos profissionais de saúde, principalmente na APS, já que foi o ponto de atenção associado com a dificuldade de acesso.

Destaca-se a importância de adotar medidas de acolhimento e educação permanente para os profissionais, no intuito de efetivar o acesso aos serviços de saúde e melhorar a qualidade da assistência prestada.

\section{Referências}

1. Silva Júnior ES, Medina MG, Aquino R, Fonseca ACF, Vilasbôas ALQ. Acessibilidade geográfica à atenção primária à saúde em distrito sanitário do município de Salvador, Bahia. Rev Bras Saude Mater Infant.2010;10(Suppl 1):s49-s60. DOI: http://dx.doi.org/10.1590/S1519-38292010000500005

2. Figueroa Pedraza D, Cavalcanti Costa GM. Accesibilidad a los servicios públicos de salud: la visión de los usuarios de la Estrategia Salud de la Familia. Enferm Glob. 2014;13(1):267-78. DOI: http://dx.doi.org/10.6018/eglobal.13.1.170901

3. Brasil. Instituto Brasileiro de Geografia e Estatística (IBGE). Pesquisa Nacional por Amostra de Domicílios - um panorama da saúde no Brasil: acesso e utilização dos serviços, condições de saúde e fatores de risco e proteção à saúde 2008. Rio de Janeiro: IBGE; 2010.

4. Jesus WLA, Assis MMA. Revisão sistemática sobre o conceito de acesso nos serviços de saúde: contribuições do planejamento. Ciênc Saúde Coletiva. 2010;15(1):161-70. DOI: http://dx.doi.org/10.1590/S1413-81232010000100022

5. Donabedian A. An introduction to quality assurance in health care. New York: Oxford University Press; 2002.

6. Travassos C, Viacava F. Acesso e uso de serviços de saúde em idosos residentes em áreas rurais, Brasil, 1998 e 2003. Cad. Saúde Pública. 2007;23(10):2490-502. DOI: http://dx.doi.org/10.1590/S0102-311X2007001000023

7. Brasil. Ministério da Saúde. Secretaria de Vigilância em Saúde. Secretaria de Gestão Estratégica e Participativa. Vigitel Brasil 2010: vigilância de fatores de risco e proteção para doenças crônicas por inquérito telefônico. Brasília: Ministério da Saúde; 2011. 152 p.

8. Dargatz DA, Hill GW. Analysis of survey data. Prev Vet Med. 1996;28(4):225-37. DOI: http://dx.doi.org/10.1016/0167-5877(96)01052-5

9. Szwarcwald CL, Damacena GN. Amostras complexas em inquéritos populacionais: planejamento e implicações na análise estatística dos dados. Rev Bras Epidemiol. 2008;11(Suppl1):38-45. DOI: http://dx.doi.org/10.1590/S1415$790 \times 2008000500004$

10. Assis MMA, Villa TCS, Nascimento MAA. Acesso aos serviços de saúde: uma possibilidade a ser construída na prática. Ciênc Saúde Coletiva. 2003;8(3):815-23. DOI: http://dx.doi.org/10.1590/S1413-81232003000300016

11. Andrade MV, Noronha K, Barbosa ACQ, Rocha TAH, Silva NC, Calazans JA, et al. A equidade na cobertura da Estratégia Saúde da Família em Minas Gerais, Brasil. Cad Saúde Pública. 2015;31(6):1175-87. DOI: http://dx.doi.org/10.1590/0102$311 \times 00130414$

12. Jusot F. Lesinégalités de recoursaux soins: bilan et évolution. Rev Epidemiol Sante Publique. 2013;61(Suppl 3):163-9. DOI: http://dx.doi.org/10.1016/j.respe.2013.05.011

13. Garcia-Subirats I, Vargas I, Mogollón-Pérez AS, De Paepe P, Silva MR, Unger JP, et al. Barriers in access to healthcare in countries with different health systems. A cross-sectional study in municipalities of central Colombia and north-eastern Brazil. Soc Sci Med. 2014;106:204-13. DOI: http://dx.doi.org/10.1016/j.socscimed.2014.01.054 
14. Reis RS, Coimbra LC, Silva AAM, Santos AM, Alves MTSSB, Lamy ZC, et al. Acesso e utilização dos serviços na Estratégia Saúde da Família na perspectiva dos gestores, profissionais e usuários. Ciênc Saúde Coletiva. 2013;18(11):3321-31. DOI: http://dx.doi.org/10.1590/S1413-81232013001100022

15. Miquilin IOC, Marín-León L, Monteiro MI, Corrêa Filho HR. Desigualdades no acesso e uso dos serviços de saúde entre trabalhadores informais e desempregados: análise da PNAD 2008, Brasil. Cad Saúde Pública. 2013;29(7):1392-406. DOI: http://dx.doi.org/10.1590/S0102-311X2013000700013

16. Brasil. Ministério da Saúde. Secretaria de Atenção à Saúde. Núcleo Técnico da Política Nacional de Humanização. Acolhimento nas práticas de produção de saúde. Brasília: Ministério da Saúde; 2010.

17. Lima MADS, Ramos DD, Rosa RB, Nauderer TM, Davis R. Acesso e acolhimento em unidades de saúde na visão dos usuários. Acta Paul Enferm. 2007;20(1):12-7. DOI: http://dx. doi.org/10.1590/S0103-21002007000100003

18. Takemoto MLS, Silva EM. Acolhimento e transformações no processo de trabalho de enfermagem em unidades básicas de saúde de Campinas, São Paulo, Brasil. Cad Saúde Pública. 2007;23(2):331-40. DOI: http://dx.doi.org/10.1590/S0102311X2007000200009

19. Silva TF, Romano VF. O acolhimento e o Núcleo de Apoio à Saúde da Família no município do Rio de Janeiro: fragmentos, perspectivas e reflexões. Rev Bras Med Fam Comunidade. 2015;10(34):1-7. DOI: http://dx.doi.org/10.5712/rbmfc10(34)1010

20. Lima SAV, Silva MRF, Carvalho EMF, Pessoa EAC, Brito ESV, Braga JPR. Elementos que influenciam o acesso à atenção primária na perspectiva dos profissionais e dos usuários de uma rede de serviços de saúde do Recife. Physis. 2015;25(2):635-56. DOI: http://dx.doi.org/10.1590/S0103-73312015000200016

21. Boing AF, Matos IB, Arruda MP, Oliveira MC, Njaine K. Prevalência de consultas médicas e fatores associados: um estudo de base populacional no sul do Brasil. Rev Assoc Med Bras. 2010;56(1):41-6. DOI: http://dx.doi.org/10.1590/S010442302010000100014

22. Dias-da-Costa JS, Gigante DP, Horta BL, Barros FC, Victora CG. Utilização de serviços de saúde por adultos da coorte de nascimentos de 1982 a 2004-5, Pelotas, RS. Rev Saúde Pública. 2008;42(Suppl 2):51-9. DOI: http://dx.doi.org/10.1590/S0034-89102008000900008

23. Pilger $\mathrm{C}$, Menon UM, Mathias TAF. Health services use among elderly people living in the community. Rev Esc Enferm USP. 2013;47(1):213-20. DOI: http://dx.doi.org/10.1590/S0080-62342013000100027

24. Barata RB. Acesso e uso de serviços de saúde. Considerações sobre os resultados da Pesquisa de Condições de Vida 2006. São Paulo Perspect. 2008;22(2):19-29.

25. Oliveira LS, Almeida LGN, Oliveira MAS, Gil GB, Cunha ABO, Medina MG, et al. Acessibilidade a atenção básica em um distrito sanitário de Salvador. Ciênc Saúde Coletiva. 2012;17(11):3047-56. DOI: http://dx.doi.org/10.1590/S141381232012001100021

26. Terraneo M. Inequities in health care utilization by people aged 50+: evidence from 12 European countries. Soc Sci Med. 2015;126:154-63. PMID: 25562311 DOI: http://dx.doi.org/10.1016/j.socscimed.2014.12.028

27. Regidor E, Martínez D, Calle ME, Astasio P, Ortega P, Domínguez V. Socioeconomic patterns in the use of public and private health services and equity in health care. BMC Health Serv Res. 2008;8:183. DOI: http://dx.doi.org/10.1186/1472-6963-8-183

28. Costa SM, Araújo FF, Martins LV, Nobre LLR, Araújo FM, Rodrigues CAQ. Agente Comunitário de Saúde: elemento nuclear das ações em saúde. Ciênc Saúde Coletiva. 2013;18(7):2147-56. DOI: http://dx.doi.org/10.1590/S141381232013000700030

29. Costa SM, Souza LPS, Souza TR, Cerqueira ALN, Botelho BL, Araújo EPP, et al. Práticas de trabalho no âmbito coletivo: profissionais da equipe Saúde da Família. Cad Saúde Coletiva. 2014;22(3):292-9. DOI: http://dx.doi.org/10.1590/1414462X201400030011 
Orlene Veloso Dias. Departamento de Enfermagem da Universidade Estadual de Montes Claros (Unimontes). Montes Claros, MG, Brasil. orlenedias@yahoo.com.br

Flávia Ferreira Araújo. Universidade Estadual de Montes Claros (Unimontes). Montes Claros, MG, Brasil. flaviamoc@gmail.com

Rayana Meireles de Oliveira. Universidade Estadual de Montes Claros (Unimontes). Montes Claros, MG, Brasil. razinhameireles@ hotmail.com

Rosângela Barbosa Chagas. Departamento de Enfermagem da Universidade Estadual de Montes Claros (Unimontes). Montes Claros, MG, Brasil. rosachagas@yahoo.com.br

Simone de Melo Costa. Departamento de Odontologia e do Mestrado Profissional em Cuidado Primário em Saúde da Universidade Estadual de Montes Claros (Unimontes). Montes Claros, MG, Brasil. smelocosta@gmail.com (Autora correspondente) 